\title{
SYNTHESIS OF BIS-IMIDAZOLONE CONDENSED RING DERIVATIVES BEARING POTENTIAL FUNGICIDAL ACTIVITIES
}

\author{
Yong Sun ${ }^{*}$, Li-Ping Gao ${ }^{a}$, Ming-Wu Ding ${ }^{b}$ \\ Department of Chemistry, Yumyang Teachers College, Danjiangkou, 442700, P.R. China ${ }^{a}$ and \\ College of Chemistry, Central China Normal University, Wuhan, 430079, P.R. China ${ }^{b}$
}

\begin{abstract}
Bis-imidazolone condensed ring derivatives $\underline{4}$ were synthesized by S-alkylation of bisimidazolidinones $\underline{\mathbf{3}}$, which were obtained via tandem aza-Wittig reaction of vinyliminophosphoranes $\underline{\mathbf{1}}$, carbon disulfide and ethylenediamine.
\end{abstract}

\section{Introduction}

4H-Imidazol-4-ones are important heterocycles having biological activities(1-3). Some derivatives of 5furfurylidene-4H-imidazolin-4-one were found to show good antiinflammatory activity(4). Some of them appear in a variety of biologically active molecules in which a common structural unit is a derivatized 2alkylthio-4H-imidazol-4-one moiety(5-7). However, most of the 2-alkylthioimidazolones reported are of the 5,5-disubstituted type and were generally synthesized from corresponding $\alpha$-amino acetic acid(7,8) (Scheme 1). Unfortunately, 5-arylmethylidene-2-alkylthioimidazolones cannot be prepared by this general method for the corresponding starting material needed would be unstable vinyl amino acetic acids. Recently, we are interested in the synthesis of biologically active imidazolones via tandem azaWittig reaction(9-15). Here we report the synthesis of bis-imidazolone condensed ring derivatives $\underline{4}$ from the stable vinyliminophosphoranes $\underline{1}$ (Scheme 2).

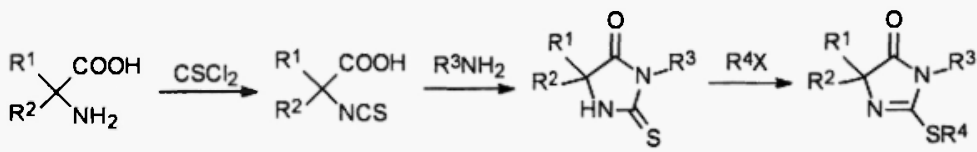

Scheme 1

Address correspondence to Yong Sun, Department of Chemistry, Yunyang Teachers College, Danjiangkou, 442700, P.R.

China. E-mail: sunyong6111@sina.com 


\section{Results and Discussion}

The easily accessible vinyliminophosphoranes $\underline{1}$ reacted with carbon disulfide to give vinyl isothiocyanates $\underline{\mathbf{2}}$. The reaction of $\underline{\mathbf{2}}$ with ethylenediamine took place smoothly at room temperature to give the yellow crystal bis-imidazolidinones $\underline{3}$ in 79 87\% yields (Scheme 2 and Table 1).

S-Alkylation of $\underline{\mathbf{3}}$ with dibromohydrocarbons in presence of solid potassium carbonate provided bisimidazolone condensed ring derivatives $\underline{4}$ in $41 \sim 62 \%$ yields. The alkylation had to be carried out at 50 60L (Scheme 2 and Table 1).

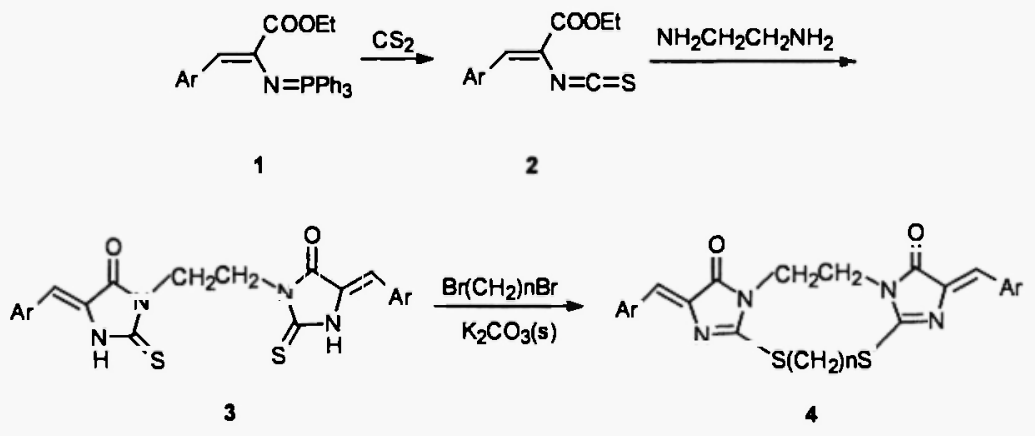

Scheme 2

The structure of $\underline{3}$ and $\underline{4}$ has been confirmed by spectral date ${ }^{1} \mathrm{H}$ NMR, IR and MS. For example, the ${ }^{1} \mathrm{H}$ NMR spectrum data in $\underline{4 b}$ show the signals of $=\mathrm{CH}$ and $-\mathrm{NCH}_{2} \mathrm{CH}_{2} \mathrm{~N}-$ at $6.98 \mathrm{ppm}$ and $3.87 \mathrm{ppm}$ as single absorption respectively. The chemical shift of aryl hydrogens is $8.15 \sim 7.26 \mathrm{ppm}$ with multiple absorption. The other signals appeared at $3.35\left(\mathrm{t}, 4 \mathrm{H}, \mathrm{SCH}_{2}\right)$ and $2.24\left(\mathrm{~m}, 2 \mathrm{H}, \mathrm{SCH}_{2} \underline{\mathrm{CH}}_{2} \mathrm{CH}_{2} \mathrm{~S}\right)$. The IR of $\underline{4 b}$ showed the strong stretching vibration peak of imidazolone $\mathrm{C}=\mathrm{O}$ at $1720 \mathrm{~cm}^{-1}$ and the peak of $\mathrm{C}=\mathrm{C}$ at about 1638 $\mathrm{cm}^{-1}$. The MS of 4 b showed $\mathrm{M}^{+}$at $\mathrm{m} / \mathrm{z} 474$ with $30 \%$ abundance. 
Table-1: Preparation of bis-imidazolidinones $\underline{3}$ and bis-imidazolone condensed ring derivatives $\underline{4}$

\begin{tabular}{cccccc}
\hline Entry & Ar & $\mathrm{n}$ & Conditoin & Yield(\%) ${ }^{\mathrm{a}}$ & $\mathrm{m} . \mathrm{p} .\left({ }^{\circ} \mathrm{C}\right)$ \\
\hline$\underline{\mathbf{3 a}}$ & $\mathrm{Ph}$ & & r.t. $/ 3 \mathrm{~h}$ & 87 & $277 \sim 278$ \\
$\underline{\mathbf{3 b}}$ & Furyl & & r.t. $/ 4 \mathrm{~h}$ & 79 & $255 \sim 257$ \\
$\underline{\mathbf{4 a}}$ & $\mathrm{Ph}$ & 2 & $60^{\circ} \mathrm{C} / 6 \mathrm{~h}$ & 41 & $161 \sim 162$ \\
$\underline{\mathbf{4 b}}$ & $\mathrm{Ph}$ & 3 & $50^{\circ} \mathrm{C} / 4 \mathrm{~h}$ & 50 & $161 \sim 163$ \\
$\underline{\mathbf{4 c}}$ & $\mathrm{Ph}$ & 4 & $50^{\circ} \mathrm{C} / 3 \mathrm{~h}$ & 57 & $161 \sim 162$ \\
$\underline{\mathbf{4 d}}$ & $\mathrm{Ph}$ & 5 & $60^{\circ} \mathrm{C} / 6 \mathrm{~h}$ & 55 & $152 \sim 154$ \\
$\underline{\mathbf{4 e}}$ & $\mathrm{Ph}$ & 6 & $60^{\circ} \mathrm{C} / 7 \mathrm{~h}$ & 45 & $145 \sim 147$ \\
$\underline{\mathbf{4 f}}$ & Furyl & 2 & $60^{\circ} \mathrm{C} / 7 \mathrm{~h}$ & 48 & $133 \sim 134$ \\
$\underline{4 \mathbf{g}}$ & Furyl & 3 & $50^{\circ} \mathrm{C} / 4 \mathrm{~h}$ & 56 & $135 \sim 136$ \\
$\underline{\mathbf{4 h}}$ & Furyl & 4 & $50^{\circ} \mathrm{C} / 4 \mathrm{~h}$ & 62 & $131 \sim 132$ \\
$\underline{\mathbf{4 i}}$ & Furyl & 5 & $60^{\circ} \mathrm{C} / 8 \mathrm{~h}$ & 59 & $138 \sim 140$ \\
$\underline{\mathbf{4 i}}$ & Furyl & 6 & $60^{\circ} \mathrm{C} / 8 \mathrm{~h}$ & 53 & $167 \sim 168$ \\
\hline
\end{tabular}

AA: Isolated yields of $\underline{3}$ based on vinyliminophosphoranes $\underline{1}$; B: Purified yields of $\underline{4}$ based on bis-imidazolidinones $\underline{3}$.

\section{Experimental}

Melting points were uncorrected. MS were measured on a Finnigan Trace spectrometer. IR were recorded on a PE-983 infrared spectrometer as $\mathrm{KBr}$ pellets with absorption in $\mathrm{cm}^{-1}$. NMR were taken in $\mathrm{CDCl}_{3}$ or DMSO- $\mathrm{d}_{6}$ on a Varian Mercury 400 spectrometer and resonances are given in ppm $(\delta)$ relative to TMS. Elementary analyses were taken on a Perkin-Elmer $2400 \mathrm{CHN}$ Elementary Analysis Instrument. $\mathrm{CS}_{2}$ is poisonous and a good hood should be used. Vinyliminophosphoranes 1 was prepared by the literature report(16).

General Preparation of Bis-imidazolidinones $\underline{3}$-To a solution of vinyliminophosphoranes 1 ( $(5 \mathrm{mmol})$ in dry methylene dichloride $(15 \mathrm{~mL})$ was added excess carbon disufide $(5 \mathrm{~mL})$. After the reaction mixture was refluxed for $28 \mathrm{~h}$, the solvent was removed under reduced pressure and ether/petroleum ether $(1: 2,20 \mathrm{~mL})$ was added to precipitate triphenylphosphine sulfide which was removed by filtration. The filtrate was evaporated to give vinyl isothiocyanates $\underline{\mathbf{2}}$, which was used directly without further purification. To the solution of $\underline{\mathbf{2}}$ in $\mathrm{CH}_{3} \mathrm{CN}(15 \mathrm{~mL})$ was added ethylenediamine $(0.17 \mathrm{~mL}, 2.5 \mathrm{mmol})$. The mixture was allowed to stand for $3 \sim 4 \mathrm{~h}$ at room temperature and the precipitated solid was collected and washed with water and ethanol, recrystallized from methylene dichloride/petroleum ether to give bis-imidazolidinones $\underline{\mathbf{3}}$. 
recrystallized from methylene dichloride/petroleum ether to give bis-imidazolidinones $\underline{\mathbf{3}}$.

3a: yellow crystals, ${ }^{1} \mathrm{H}$ NMR(DMSO- $\left.\mathrm{d}_{6}, 400 \mathrm{MHz}\right)[]$ 9.06(s, $\left.2 \mathrm{H}, \mathrm{N}-\mathrm{H}\right), 7.79 \sim 7.40(\mathrm{~m}, 10 \mathrm{H}, \mathrm{Ph}-\mathrm{H})$, 6.55(s, $2 \mathrm{H},=\mathrm{CH}), 4.14\left(\mathrm{~s}, 4 \mathrm{H}, \mathrm{CH}_{2} \mathrm{CH}_{2}\right) ; \operatorname{IR}\left(\mathrm{cm}^{-1}\right), 3255(\mathrm{~N}-\mathrm{H}), 1718(\mathrm{C}=\mathrm{O}), 1646(\mathrm{C}=\mathrm{C}) ; \mathrm{MS}(\mathrm{m} / \mathrm{z}, \%)$, 434( $\left.\mathrm{M}^{+}, 88\right), 401(2)$, 230(49), 204(32), 188(12), 160(39), 144(7), 117(100), 102(13), 89(43); Anal. Calcd. for $\mathrm{C}_{22} \mathrm{H}_{18} \mathrm{~N}_{4} \mathrm{O}_{2} \mathrm{~S}_{2}$ : C, 60.83; H, 4.15; N, 12.90. Found: $\mathrm{C}, 61.09 ; \mathrm{H}, 3.98 ; \mathrm{N}, 13.14$.

3b: yellow crystals, ${ }^{1} \mathrm{H}$ NMR(DMSO-d $\left.d_{6}, 400 \mathrm{MHz}\right) \square 9.30(\mathrm{~s}, 2 \mathrm{H}, \mathrm{N}-\mathrm{H}), 7.82 \sim 6.41(\mathrm{~m}, 6 \mathrm{H}, \mathrm{Ar}-\mathrm{H})$, 6.23(s, $2 \mathrm{H},=\mathrm{CH}), 4.08\left(\mathrm{~s}, 4 \mathrm{H}, \mathrm{CH}_{2} \mathrm{CH}_{2}\right) ; \mathrm{IR}\left(\mathrm{cm}^{-1}\right), 3324(\mathrm{~N}-\mathrm{H}), 1707(\mathrm{C}=\mathrm{O}), 1650(\mathrm{C}=\mathrm{C}) ; \mathrm{MS}(\mathrm{m} / \mathrm{z}, \%)$, 414(M+10), 373(8), 365(18), 290(5), 220(11), 194(10), 169(19), 107(22), 77(22), 40(100). Anal. Calcd. for $\mathrm{C}_{18} \mathrm{H}_{14} \mathrm{~N}_{4} \mathrm{O}_{4} \mathrm{~S}_{2}: \mathrm{C}, 52.17 ; \mathrm{H}, 3.38 ; \mathrm{N}, 13.53$. Found: $\mathrm{C}, 51.95 ; \mathrm{H}, 3.11 ; \mathrm{N}, 13.82$.

Preparation of Bis-imidazolone Condensed Ring Derivatives 4-A mixture of $\underline{\mathbf{3}}(4 \mathrm{mmol})$, dibromohydrocarbons (4mmol) and solid potassium carbonate $(1.11 \mathrm{~g}, 8 \mathrm{mmol})$ in $\mathrm{CH}_{3} \mathrm{CN}(30 \mathrm{~mL})$ was stirred for $3 \sim 8 \mathrm{~h}$ at $50 \sim 60$ and filtered, the filtrate was condensed and the residual was recrystallized from methylene dichloride/petroleum ether to give bis-imidazolone condensed ring derivatives $\underline{4}$

4a: yellow crystals, ${ }^{1} \mathrm{H}$ NMR( $\left.\mathrm{CDCl}_{3}, 400 \mathrm{MHz}\right)[18.15 \sim 7.26(\mathrm{~m}, 10 \mathrm{H}, \mathrm{Ph}-\mathrm{H}), 6.98(\mathrm{~s}, 2 \mathrm{H},=\mathrm{CH}), 3.85(\mathrm{~s}$, $\left.4 \mathrm{H}, \mathrm{NCH}_{2} \mathrm{CH}_{2} \mathrm{~N}\right), 3.38\left(\mathrm{~s}, 4 \mathrm{H}, \mathrm{SCH}_{2} \mathrm{CH}_{2} \mathrm{~S}\right) ; \mathrm{IR}\left(\mathrm{cm}^{-1}\right), 1718(\mathrm{C}=\mathrm{O}), 1637(\mathrm{C}=\mathrm{C}) ; \mathrm{MS}(\mathrm{m} / \mathrm{z}, \%), 460\left(\mathrm{M}^{+}, 1\right)$, 434(2), 314(1), 288(2), 231(5), 203(6), 188(6), 160(25), 144(11), 130(14), 121(31), 116(100), 91(87); Anal. Calcd. for $\mathrm{C}_{24} \mathrm{H}_{20} \mathrm{~N}_{4} \mathrm{O}_{2} \mathrm{~S}_{2}: \mathrm{C}, 62.61 ; \mathrm{H}, 4.35 ; \mathrm{N}, 12.17$. Found: C, 62.88; H, 4.57; N, 12.44.

4b: yellow crystals, ${ }^{1} \mathrm{H}$ NMR( $\left(\mathrm{CDCl}_{3}, 400 \mathrm{MHz}\right) \square 8.15 \sim 7.26(\mathrm{~m}, 10 \mathrm{H}, \mathrm{Ph}-\mathrm{H}), 6.98(\mathrm{~s}, 2 \mathrm{H},=\mathrm{CH}), 3.87(\mathrm{~s}$, $\left.4 \mathrm{H}, \mathrm{NCH}_{2} \mathrm{CH}_{2} \mathrm{~N}\right), 3.35\left(\mathrm{t}, 4 \mathrm{H}, \mathrm{SCH}_{2}\right), 2.24\left(\mathrm{~m}, 2 \mathrm{H}, \mathrm{SCH}_{2} \mathrm{CH}_{2} \mathrm{CH}_{2} \mathrm{~S}\right): \operatorname{IR}\left(\mathrm{cm}^{-1}\right), 1720(\mathrm{C}=\mathrm{O}), 1638(\mathrm{C}=\mathrm{C})$; MS (m/z, \%), $474\left(\mathrm{M}^{+}, 30\right), 459(4), 445(5), 414(6), 271(100), 255(15), 244(44), 230(33), 202(22), 129(75)$, 116(94), 91(87); Anal. Calcd. for $\mathrm{C}_{25} \mathrm{H}_{22} \mathrm{~N}_{4} \mathrm{O}_{2} \mathrm{~S}_{2}$ : C, 63.29; H, 4.64; N, 11.81. Found: C, 63.52; H, 4.79; N, 12.06 .

4c: yellow crystals, ${ }^{1} \mathrm{H}$ NMR(CDCl $\left.3,400 \mathrm{MHz}\right)|| 8.15 \sim 7.26(\mathrm{~m}, 10 \mathrm{H}, \mathrm{Ph}-\mathrm{H}), 6.97(\mathrm{~s}, 2 \mathrm{H},=\mathrm{CH}), 3.86(\mathrm{~s}$, $\left.4 \mathrm{H}, \mathrm{NCH}_{2} \mathrm{CH}_{2} \mathrm{~N}\right), 3.20\left(\mathrm{t}, 4 \mathrm{H}, \mathrm{SCH}_{2}\right), 1.82\left(\mathrm{~m}, 4 \mathrm{H}, \mathrm{SCH}_{2} \mathrm{CH}_{2} \mathrm{CH}_{2} \mathrm{CH}_{3} \mathrm{~S}\right): \operatorname{IR}\left(\mathrm{cm}^{-1}\right), 1722(\mathrm{C}=\mathrm{O}), 1640(\mathrm{C}=\mathrm{C})$; MS (m/z, \%), $489\left(\mathrm{M}^{+}+1,3\right), 344(2), 285(6), 243(1), 231(5), 203(5), 160(11), 144(7), 130(26), 116(37)$, 91(27), 55(100); Anal. Calcd. for $\mathrm{C}_{26} \mathrm{H}_{24} \mathrm{~N}_{4} \mathrm{O}_{2} \mathrm{~S}_{2}$ : C, 63.93; H, 4.92; N, 11.48. Found: C, 64.19; H, 5.17; N, 11.76 .

4d: yellow crystals, ${ }^{1} \mathrm{H}$ NMR( $\left.\mathrm{CDCl}_{3}, 400 \mathrm{MHz}\right)[8.14 \sim 7.26(\mathrm{~m}, 10 \mathrm{H}, \mathrm{Ph}-\mathrm{H}), 6.96(\mathrm{~s}, 2 \mathrm{H},=\mathrm{CH}), 3.86(\mathrm{~s}$, $\left.4 \mathrm{H}, \quad \mathrm{NCH}_{2} \mathrm{CH}_{2} \mathrm{~N}\right), 3.20$ (t, $\left.4 \mathrm{H}, \mathrm{SCH}_{2}\right), 1.67$ (m, 4H, SCH $\left.2 \mathrm{CH}_{2} \mathrm{CH}_{2} \mathrm{CH}_{2} \mathrm{CH}_{3} \mathrm{~S}\right), 1.43$ (m, 2H, 
$\left.\mathrm{SCH}_{2} \mathrm{CH}_{2} \mathrm{CH}_{2} \mathrm{CH}_{2} \mathrm{CH}_{2} \mathrm{~S}\right) ; \mathrm{IR}\left(\mathrm{cm}^{-1}\right), 1722(\mathrm{C}=\mathrm{O}), 1640(\mathrm{C}=\mathrm{C}) ; \mathrm{MS}(\mathrm{m} / \mathrm{z}, \%), 503\left(\mathrm{M}^{+}+1,8\right), 469(3), 434(6)$, 401(4), 299(5), 231(20), 203(10), 160(32), 144(16), 116(91), 89(32), 69(83), 41(100); Anal. Calcd. for $\mathrm{C}_{27} \mathrm{H}_{26} \mathrm{~N}_{4} \mathrm{O}_{2} \mathrm{~S}_{2}: \mathrm{C}, 64.54 ; \mathrm{H}, 5.18 ; \mathrm{N}, 11.16$. Found: C, 64.80; H, 5.19; N, 11.21 .

4e : yellow crystals, ${ }^{1} \mathrm{H}$ NMR( $\left.\mathrm{CDCl}_{3}, 400 \mathrm{MHz}\right) \square 8.15 \sim 7.26(\mathrm{~m}, 10 \mathrm{H}, \mathrm{Ph}-\mathrm{H}), 6.96(\mathrm{~s}, 2 \mathrm{H},=\mathrm{CH}), 3.87(\mathrm{~s}$, $\left.4 \mathrm{H}, \mathrm{NCH}_{2} \mathrm{CH}_{2} \mathrm{~N}\right), 3.24\left(\mathrm{t}, 4 \mathrm{H}, \mathrm{SCH}_{2}\right), 1.67$ (m, 4H, SCH $\left.\mathrm{CH}_{2} \mathrm{CH}_{2} \mathrm{CH}_{2} \mathrm{CH}_{2} \mathrm{CH}_{2} \mathrm{~S}\right), 1.30$ (m, 4H, $\left.\mathrm{SCH}_{2} \mathrm{CH}_{2} \mathrm{CH}_{2} \mathrm{CH}_{2} \mathrm{CH}_{2} \mathrm{CH}_{2} \mathrm{~S}\right) ; \mathrm{IR}\left(\mathrm{cm}^{-1}\right), 1721(\mathrm{C}=\mathrm{O}), 1640(\mathrm{C}=\mathrm{C}) ; \mathrm{MS}(\mathrm{m} / \mathrm{z}, \%), 517\left(\mathrm{M}^{+}+1,17\right), 503(3)$, 483(2), 434(16), 401(6), 369(9), 313(6), 231(36), 203(14), 160(45), 144(19), 116(65), 55(100); Anal. Calcd. for $\mathrm{C}_{28} \mathrm{H}_{28} \mathrm{~N}_{4} \mathrm{O}_{2} \mathrm{~S}_{2}: \mathrm{C}, 65.12 ; \mathrm{H}, 5.43 ; \mathrm{N}, 10.85$. Found: $\mathrm{C}, 64.89 ; \mathrm{H}, 5.68 ; \mathrm{N}, 11.13$.

4f: yellow crystals, ${ }^{1} \mathrm{H}$ NMR( $\left(\mathrm{CDCl}_{3}, 400 \mathrm{MHz}\right) \square 7.58 \sim 6.92(\mathrm{~m}, 6 \mathrm{H}, \mathrm{Ar}-\mathrm{H}), 6.58(\mathrm{~s}, 2 \mathrm{H},=\mathrm{CH}), 3.84(\mathrm{~s}, 4 \mathrm{H}$, $\left.\mathrm{NCH}_{2} \mathrm{CH}_{2} \mathrm{~N}\right), 3.23\left(\mathrm{~s}, 4 \mathrm{H}, \mathrm{SCH}_{2}\right) ; \mathrm{IR}\left(\mathrm{cm}^{-1}\right), 1723(\mathrm{C}=\mathrm{O}), 1643(\mathrm{C}=\mathrm{C}) ; \mathrm{MS}(\mathrm{m} / \mathrm{z}, \%), 441\left(\mathrm{M}^{+}+1,6\right), 306(5)$, 248(39), 221(58), 192(18), 178(11), 160(16), 150(21), 134(14), 120(49), 107(100), 92(17); Anal. Calcd. for $\mathrm{C}_{20} \mathrm{H}_{16} \mathrm{~N}_{4} \mathrm{O}_{4} \mathrm{~S}_{2}: \mathrm{C}, 54.54 ; \mathrm{H}, 3.64 ; \mathrm{N}, 12.73$. Found: $\mathrm{C}, 54.78 ; \mathrm{H}, 3.81 ; \mathrm{N}, 13.00$.

4g: yellow crystals, ${ }^{1} \mathrm{H}$ NMR( $\left(\mathrm{CDCl}_{3}, 400 \mathrm{MHz}\right) \square 7.60 \sim 6.91(\mathrm{~m}, 6 \mathrm{H}, \mathrm{Ar}-\mathrm{H}), 6.58(\mathrm{~s}, 2 \mathrm{H},=\mathrm{CH}), 3.85(\mathrm{~s}$, $\left.4 \mathrm{H}, \mathrm{NCH}_{2} \mathrm{CH}_{2} \mathrm{~N}\right), 3.24\left(\mathrm{t}, 4 \mathrm{H}, \mathrm{SCH}_{2}\right), 1.87\left(\mathrm{~m}, 2 \mathrm{H}, \mathrm{SCH}_{2} \mathrm{CH}_{2} \mathrm{CH}_{3} \mathrm{~S}\right): \operatorname{IR}\left(\mathrm{cm}^{-1}\right), 1722(\mathrm{C}=\mathrm{O}), 1641(\mathrm{C}=\mathrm{C}) ; \mathrm{MS}$ (m/z, \%), $455\left(\mathrm{M}^{+}+1,4\right), 429(3), 320(4), 262(47), 235(12), 220(38), 193(13), 178(17), 161(13), 149(25)$, 134(11), 120(46), 107(49), 92(10), 81(14), 41(100); Anal. Calcd. for $\mathrm{C}_{21} \mathrm{H}_{18} \mathrm{~N}_{4} \mathrm{O}_{4} \mathrm{~S}_{2}: \mathrm{C}, 55.51 ; \mathrm{H}, 3.96 ; \mathrm{N}$, 12.33. Found: C, $55.77 ; \mathrm{H}, 4.19 ; \mathrm{N}, 12.58$.

4h: yellow crystals, ${ }^{1} \mathrm{H}$ NMR( $\left.\mathrm{CDCl}_{3}, 400 \mathrm{MHz}\right)$ 7.59 6.92 (m, 6H, Ar-H), $6.58(\mathrm{~s}, 2 \mathrm{H},=\mathrm{CH}), 3.84(\mathrm{~s}$, $\left.4 \mathrm{H}, \mathrm{NCH}_{2} \mathrm{CH}_{2} \mathrm{~N}\right), 3.26\left(\mathrm{t}, 4 \mathrm{H}, \mathrm{SCH}_{2}\right), 1.86\left(\mathrm{~m}, 4 \mathrm{H}, \mathrm{SCH}_{2} \mathrm{CH}_{2} \mathrm{CH}_{2} \mathrm{CH}_{2} \mathrm{~S}\right) ; \mathrm{IR}\left(\mathrm{cm}^{-1}\right), 1716(\mathrm{C}=\mathrm{O}), 1634(\mathrm{C}=\mathrm{C})$; MS (m/z, \%), $469\left(\mathrm{M}^{+}+1,5\right), 443(3), 334(3), 275(59), 249(17), 233(4), 221(32), 194(17), 178(12), 162(10)$, 150(30), 134(17), 120(51), 107(76), 92(13), 81(35), 55(100); Anal. Calcd. for $\mathrm{C}_{22} \mathrm{H}_{20} \mathrm{~N}_{4} \mathrm{O}_{4} \mathrm{~S}_{2}: \mathrm{C}, 56.41 ; \mathrm{H}$, $4.27 ; \mathrm{N}, 11.97$. Found: C, $56.66 ; \mathrm{H}, 4.40 ; \mathrm{N}, 12.21$.

4i: yellow crystals, ${ }^{1} \mathrm{H}$ NMR( $\left.\mathrm{CDCl}_{3}, 400 \mathrm{MHz}\right) \square 7.58 \sim 6.91(\mathrm{~m}, 6 \mathrm{H}, \mathrm{Ar}-\mathrm{H}), 6.58(\mathrm{~s}, 2 \mathrm{H},=\mathrm{CH}), 3.84(\mathrm{~s}, 4 \mathrm{H}$, $\left.\mathrm{NCH}_{2} \mathrm{CH}_{2} \mathrm{~N}\right), \quad 3.25$ (t, $\left.4 \mathrm{H}, \quad \mathrm{SCH}_{2}\right), \quad 1.72\left(\mathrm{~m}, \quad 4 \mathrm{H}, \quad \mathrm{SCH}, \underline{\mathrm{CH}}_{2} \mathrm{CH}_{2} \underline{\mathrm{CH}}_{2} \mathrm{CH}_{2} \mathrm{~S}\right), 1.47$ (m, $2 \mathrm{H}$, $\left.\mathrm{SCH}_{2} \mathrm{CH}_{2} \mathrm{CH}_{2} \mathrm{CH}_{2} \mathrm{CH}_{2} \mathrm{~S}\right) ; \mathrm{IR}\left(\mathrm{cm}^{-1}\right), 1717(\mathrm{C}=\mathrm{O}), 1635(\mathrm{C}=\mathrm{C}) ; \mathrm{MS}(\mathrm{m} / \mathrm{z}, \%), 483\left(\mathrm{M}^{+}+1,8\right), 449(3), 414(7)$, 381(3), 363(2), 289(7), 263(5), 247(2), 220(33), 194(18), 178(14), 150(40), 134(9), 122(20), 107(67), 69(100), 41(92); Anal. Calcd. for $\mathrm{C}_{23} \mathrm{H}_{22} \mathrm{~N}_{4} \mathrm{O}_{4} \mathrm{~S}_{2}$ : C, 57.26; H, 4.56; N, 11.62. Found: C, 57.50; H, 4.51; N, 11.89 .

4i: yellow crystals, ${ }^{\prime} \mathrm{H} \mathrm{NMR}\left(\mathrm{CDCl}_{3}, 400 \mathrm{MHz}\right) \quad 7.58 \sim 6.90(\mathrm{~m}, 6 \mathrm{H}, \mathrm{Ar}-\mathrm{H}), 6.58(\mathrm{~s}, 2 \mathrm{H},=\mathrm{CH}), 3.89(\mathrm{~s}, 4 \mathrm{H}$, 
$\left.\mathrm{NCH}_{2} \mathrm{CH}_{2} \mathrm{~N}\right), \quad 3.21$ (t, $\left.4 \mathrm{H}, \quad \mathrm{SCH}_{2}\right), 1.73\left(\mathrm{~m}, 4 \mathrm{H}, \quad \mathrm{SCH}_{2} \mathrm{CH}_{2} \mathrm{CH}_{2} \mathrm{CH}_{3} \mathrm{CH}_{2} \mathrm{CH}, \mathrm{S}\right) .1 .38$ (m, 4H, $\left.\mathrm{SCH}_{2} \mathrm{CH}_{2} \mathrm{CH}_{2} \mathrm{CH}_{2} \mathrm{CH}_{2} \mathrm{CH}_{2} \mathrm{~S}\right)$; IR( $\left(\mathrm{cm}^{-1}\right), 1719(\mathrm{C}=\mathrm{O}), 1638$ (C=C); MS (m/z,\%), $497\left(\mathrm{M}^{+}+1,5\right), 414(6)$, 381(2), 349(3), 236(3), 221(13), 194(10), 180(9), 150(20), 134(6), 122(12), 106(44), 81(19), 67(9), 55(98), 51(15), 41(100); Anal. Calcd. for $\mathrm{C}_{24} \mathrm{H}_{24} \mathrm{~N}_{4} \mathrm{O}_{4} \mathrm{~S}_{2}$ : C, 58.06; H, 4.84; N, 11.29. Found: C, 57.92; H, 5.11; N, 11.55 .

\section{Acknowledgements}

We gratefully acknowledge financial support of this work by the National Natural Science Foundation of China (Project No. 20102001) and the Natural Science Foundation of Hubei Education Commission of China (Project No. 2003A002).

\section{References}

(1) T. S. Sulkowski, D. P. Strike, H. M. Elockdah, US 5599829(1997).

(2) B. L. Pilkington, S. E. Russell, A. J. Whittle, W. R. Mound, M. D. Turnbull, A. M. Kozakiewicz, W. G. Whittingham, GB 2329180(1999).

(3) A. I. Khodair, H. I. El-Subbagh, A. M. Al-Obaid, Phosphorus, Sulfur Silicon Relat. Elem., 140, 159(1998).

(4) S. Saxena, M. Verma, A. K. Saxena, K. Shanker, Indian J. Heterocycl. Chem. 1, 34(1991).

(5) G. Emeric, J. Hutt, J. Perez, WO 9602538(1996).

(6) J. P. Bascou, A. Gadras, J. Perez, G. Emeric, G. Lacroix, C. Veyrat, EP 668270(1995).

(7) G. Lacroix, R. Peignier, R. Pepin, J. P. Bascou, J. Perez, C. Schmitz, US 6002016(1999).

(8) J. P. Bascou, G. Lacroix, A. Gadras, J. Perez, EP 629616(1994).

(9) M. W. Ding, Y. Sun, S. J. Yang, X. P. Liu, Z. J. Liu, Synth. Commun., 33, 1651 (2003).

(10) Y. Sun, M. W. Ding, Phosphorus Sulfur and Silicon, 178, 2137 (2003).

(11) Y. Sun, M. W. Ding, Heteroatom Chem., 14, 348(2003).

(12) Y. Sun, M. W. Ding, Z. J. Liu, Chin. J. Org. Chem., 23, 818(2003).

(13) M. W. Ding, S. J. Yang, Y. Sun, Z. J. Liu, X. P. Liu, Heterocycl. Commun, 8, 493 (2002).

(14) M. W. Ding, Y. Sun, X. P. Liu, Z. J. Liu, Heterocycl. Commun., 9, 135 (2003).

(15) M. W. Ding, Y. Sun, X. P. Liu, Z. J. Liu, Chinese J. Chem., 21, 577 (2003).

(16) P. Molina, P. M. Fresneda, F. Hurtado, Synthesis, 45(1987).

\section{Received on June 23, 2004}

